\title{
ON THE BOUNDARY VALUE PROBLEMS OF LINEAR ORDINARY
}

\section{DIFFERENTIAL EQUATIONS OF SECOND ORDER*}

BY

\section{MAX MASON}

It is the purpose of this paper to investigate the solutions of linear differential equations of second order which satisfy linear boundary conditions of a general form. In the first section theorems regarding the existence of solutions of the differential equation

$$
y^{\prime \prime}+p(x) y^{\prime}+q(x) y=f(x)
$$

under the boundary conditions

$$
\begin{aligned}
& a_{1} y\left(x_{1}\right)+a_{2} y\left(x_{2}\right)+a_{3} y^{\prime}\left(x_{1}\right)+a_{4} y^{\prime}\left(x_{2}\right)=A, \\
& b_{1} y\left(x_{1}\right)+b_{2} y\left(x_{2}\right)+b_{3} y^{\prime}\left(x_{1}\right)+b_{4} y^{\prime}\left(x_{2}\right)=B,
\end{aligned}
$$

will be established, under the assumption that the relation

$$
a_{2} b_{4}-a_{4} b_{2}=e^{\int_{x_{1}}^{x_{2}} p d x}\left(a_{1} b_{3}-a_{3} b_{1}\right)
$$

is satisfied. $\dagger$ These results will be derived from the general solution of the differential equation. In the following sections the differential equation

$$
y^{\prime \prime}+p(x) y^{\prime}+[\lambda A(x)-B(x)] y=0
$$

will be considered, where $\lambda$ is a parameter. The existence of functions satisfying the differential equation and the homogeneous form of the above boundary conditions for particular values of $\lambda$, i. e., the existence of normal functions for the problem, will be proved by means of certain minimum problems, based upon that used by WeBER $\ddagger$ whereby reasoning similar to that of DIRICHLET's prin-

\footnotetext{
* Presented to the Society at the Williamstown summer meeting, September 8, 1905. Received for publication February 26, 1906.

$\dagger$ 'The problem might be called in this case a self adjoint boundary problem. The nature of the problem would be considerably different if this relation were not satisfied.

$\ddagger$ Mathematische Annalen, vol. 1 (1869), p. 1.
} 
ciple was employed. By this method the properties of the normal functions as minimum functions are set in evidence, properties upon which the proof of oscillation theorems for the normal functions may be based. As an illustration an oscillation theorem for periodic solutions will be proved in the last section.

Special cases of the above boundary conditions have formed the subject of many investigations since the famous memoirs of STURM and Liouville.* Picard $\dagger$ has applied the method of successive approximations to the case $y\left(r_{1}\right)=0, y\left(x_{2}\right)=0$, for the differential equation

$$
y^{\prime \prime}+\lambda \Lambda(x) y=0,
$$

where the function $A$ does not change sign. The writer $\ddagger$ has treated certain boundary conditions for this differential equation by reducing the problem to the solution of an integral equation by means of one-dimensional Green's functions, and applying a method to which the method of $\S 2$ of the present paper is analogous. In particular the existence of periodic solutions was thus shown. These results were later reproduced by HoLmGren, $\S$ using a method based upon Hilbert's treatment of Dirichlet's principle, and by Tzitzfica, \| who used a generalization of PICARD's method. The existence of periodic solutions was also proved by Bôcher, $\uparrow$ the proof being based on STURM's results.

Important results have recently been obtained by HILBERT regarding the solution of integral equations with symmetrical Kern.** These results may however be applied to the boundary problem of the differential equation only in case the function $A(x)$ does not change sign, a restriction which will not be made in the present article. The expansion of a function in terms of normal functions, which is the result of greatest interest in HILBERT's work, has also been treated by Dixos $\dagger \dagger$ for a general boundary condition in connection with a differential equation to which the one here considered may be reduced if $A(x)$ does not change sign.

\section{§1. The existence of solutions of the general problem.}

It is required to determine a solution of the differential equation $¥ \ddagger$

* Journal de Mathématiques, vols. 1, 2 (1836, 1837). See Bôcher, Encyklopädie der mathematischen Wissenschaften, II A $7 \mathrm{a}$.

† See his Traité d'analyse, 3 (1896), p. 105 ff.

† Mathematische Annalen, vol. 58 (1904), p. 528.

§Arkiv för Matematik, Astronomi och Fysik, vol. 1 (1904), p. 401.

II Comptes rendus, vol. 140 (1905), pp. 223, 492.

II Ibid., vol. 140 (1905), p. 928.

** Nachrichten der K. Gesellschaft der Wissenschaften zu Göttingen, 1904. See also Wrstfall, Dissertation, Göttingen, 1905, and Schmidt, Dissertation, Göttingen, 1905.

††Proceedings of the London Mathematical Society, ser. 2, vol. 3 (1905), p. 83.

¥t The coefficients of the different equations considered are assumed to be continuous functions in the interval $\left(x_{1}, x_{2}\right)$. 


$$
y^{\prime \prime}+p(x) y^{\prime}+q(x) y=f(x)
$$

which satisfies the boundary conditions

$$
\left\{\begin{array}{l}
L_{a}(y) \equiv a_{1} y\left(x_{1}\right)+a_{2} y\left(x_{2}\right)+a_{3} y^{\prime}\left(x_{1}\right)+a_{4} y^{\prime}\left(x_{2}\right)=A, \\
L_{b}(y) \equiv b_{1} y\left(x_{1}\right)+b_{2} y\left(x_{2}\right)+b_{3} y^{\prime}\left(x_{1}\right)+b_{4} y^{\prime}\left(x_{2}\right)=B,
\end{array}\right.
$$

where $A, B, a_{i}, b_{i}$ are given constants. It will be assumed that the coefficients of the boundary conditions satisfy the relation

or, in writing

$$
a_{2} b_{4}-a_{4} b_{2}=e^{\int_{x_{1}}^{x_{2}} p d x}\left(a_{1} b_{3}-a_{3} b_{1}\right),
$$

$$
a_{i} b_{k}-a_{k} b_{i}=d_{i k}, \quad e^{\int_{x_{1}}^{x_{i}} p d x}=\pi,
$$

that the equation holds :

$$
d_{24}=\pi d_{13}
$$

The general solution of equation (1) is

$$
y=c_{1} u+c_{2} v+u \int_{x_{1}}^{x} f P v d x+v \int_{x}^{x_{2}} f P u d x
$$

where

$$
P=e^{\int_{x_{1}}^{x} p d x},
$$

$c_{1}$ and $c_{2}$ are arbitrary constants, and $u, v$ are linearly independent solutions of the differential equation

$$
y^{\prime \prime}+p(x) y^{\prime}+q(x) y=0,
$$

and are connected by the relation

$$
u^{\prime} v-v^{\prime} u=e^{-\int_{x_{1}}^{x} p d x}=\frac{1}{P} .
$$

The constants $c_{1}, c_{2}$ are to be so determined that $y$ satisfies the boundary conditions (2). Substituting ( 4 ) in (2), writing $v\left(x_{1}\right)=v_{1}, v^{\prime}\left(x_{1}\right)=v_{1}^{\prime}$, etc., and arranging terms, we have :

$$
\left\{\begin{array}{l}
c_{1} L_{a}(u)+c_{2} L_{a}(v)=A-\left(a_{1} v_{1}+a_{3} v_{1}^{\prime}\right) \int_{x_{1}}^{x_{2}} f P u d x-\left(a_{2} u_{2}+a_{4} u_{2}^{\prime}\right) \int_{x_{1}}^{x_{3}} f P v d x \\
c_{1} L_{b}(u)+c_{2} L_{b}(v)=B-\left(b_{1} v_{1}+b_{3} v_{1}^{\prime}\right) \int_{x_{1}}^{x_{2}} f P u d x-\left(b_{2} u_{2}+b_{4} u_{2}^{\prime}\right) \int_{x_{1}}^{x_{2}} f P v d x
\end{array}\right.
$$

If in place of $u, v$ any other pair of linearly independent solutions 


$$
\begin{aligned}
& U=d_{1} u+e_{1} v, \\
& V=d_{2} u+e_{2} v
\end{aligned}
$$

is used, the determinant $\Delta(u, v)$ of the first members of $(6)$ will be replaced by

$$
\Delta(U, V)=\left(d_{1} e_{2}-e_{1} d_{2}\right) \Delta(u, v) .
$$

Then the following theorem holds;

If $\Delta \neq 0$ there exists a unique solution of the differential equation (1) under the boundary condition (2). There exists a solution of the homogeneous problem, $i$. e., a solution not identically zero of the differential equation (1 $\left.\mathbf{1}_{0}\right)$ under the boundary conditions

when and only when $\Delta=0$.

$$
\left\{\begin{array}{l}
L_{a}(y)=0, \\
L_{b}(y)=0
\end{array}\right.
$$

Certain conditions must be satisfied by $A, B, f(x)$ in order that a solution of the non-homogeneous problem may exist when $\Delta=0$. These conditions will be different according as the minors of $\Delta$ are or are not zero. The discussion of these two cases will be given separately.

Case $I$. The minors of $\Delta$ are not all zero.

In this case but one solution of the homogeneous problem exists, except for an arbitrary constant factor. Let $u$ be this solution. Then $v$ will not be a solution, i. e., $L_{a}(v)$ and $L_{b}(v)$ are not both zero. Since $u$ satisfies $\left(2_{0}\right)$ it will also satisfy the equations :

$$
\begin{aligned}
& b_{1} L_{a}(u)-a_{1} L_{b}(u) \equiv d_{21} u\left(x_{2}\right)+d_{31} u^{\prime}\left(x_{1}\right)+d_{41} u^{\prime}\left(x_{2}\right)=0 \\
& b_{2} L_{a}(u)-a_{2} L_{b}(u) \equiv d_{12} u\left(x_{1}\right)+d_{52} u^{\prime}\left(x_{1}\right)+\pi d_{31} u^{\prime}\left(x_{2}\right)=0 \\
& b_{3} L_{a}(u)-a_{3} L_{b}(u) \equiv d_{13} u\left(x_{1}\right)+d_{23} u\left(x_{2}\right)+d_{43} u^{\prime}\left(x_{2}\right)=0 \\
& b_{4} L_{a}(u)-b_{4} L_{b}(u) \equiv d_{14} u\left(x_{1}\right)+\pi d_{13} u\left(x_{2}\right)+d_{34} u^{\prime}\left(x_{1}\right)=0
\end{aligned}
$$

The necessary and sufficient condition for the existence of a solution of (1) and (2) is from (6):

$$
\begin{aligned}
& L_{a}(v)\left\{B-\left(b_{1} v_{1}+b_{3} v_{1}^{\prime}\right) \int_{x_{1}}^{x_{3}} f P u d x-\left(b_{2} u_{2}+b_{4} u_{2}^{\prime}\right) \int_{x_{1}}^{x_{2}} f P v d x\right\} \\
& -L_{b}(v)\left\{A-\left(a_{1} v_{1}+a_{3} v_{1}^{\prime}\right) \int_{x_{1}}^{x_{2}} f P u d x-\left(a_{2} u_{2}+a_{4} u_{2}^{\prime} \int_{x_{1}}^{x_{2}} f P v d x\right\}=0 .\right.
\end{aligned}
$$

Since $u$ satisfies the boundary condition $\left(\dot{2}_{0}\right)$ the coefficient of the integral involving $v$ takes the form: 
$\left(a_{1} v_{1}+a_{3} v_{1}^{\prime}\right)\left(b_{1} u_{1}+b_{3} u_{1}^{\prime}\right)-\left(a_{2} v_{2}+a_{4} v_{2}^{\prime}\right)\left(b_{2} u_{2}+b_{4} u_{2}^{\prime}\right)$

$$
-\left(b_{1} v_{1}+b_{3} v_{1}^{\prime}\right)\left(a_{1} u_{1}+a_{3} u_{1}^{\prime}\right)+\left(b_{2} v_{2}+b_{4} v_{2}^{\prime}\right)\left(a_{2} u_{2}+a_{1} u_{2}^{\prime}\right)
$$

From (3) and (5) this reduces to

$$
=d_{13}\left(v_{1} u_{1}^{\prime}-u_{1} v_{1}^{\prime}\right)-d_{24}\left(v_{2} u_{2}^{\prime}-u_{2} v_{2}^{\prime}\right) \text {. }
$$

$$
d_{13}-d_{24} e^{-\int_{x_{1}}^{x_{2} p d x}}=d_{13}-\frac{d_{24}}{\pi}=0,
$$

and the above condition becomes :

$$
\begin{aligned}
L_{a}(v)\left\{B-\left(b_{1} v_{1}\right.\right. & \left.\left.+b_{3} v_{1}^{\prime}\right) \int_{x_{1}}^{x_{1}} f P u d x\right\} \\
& -L_{b}(v)\left\{A-\left(a_{1} v_{1}+a_{3} v_{1}^{\prime}\right) \int_{x_{1}}^{x_{2}} f P u d x\right\}=0 .
\end{aligned}
$$

Now it may be shown by means of equations (3), (5) and (7) that the following equations hold :

$$
\begin{aligned}
& L_{a}(v)\left(b_{2} u_{1}^{\prime}+\pi b_{1} u_{2}^{\prime}\right)-L_{b}(v)\left(a_{2} u_{1}^{\prime}+\pi a_{1} u_{2}^{\prime}\right)=0, \\
& L_{a}(v)\left(b_{1} u_{1}+\pi b_{3} u_{2}\right)-L_{b}(v)\left(a_{1} u_{1}-\pi a_{3} u_{2}\right)=0, \\
& L_{a}(v)\left(\pi b_{3} u_{2}^{\prime}-b_{2} u_{1}\right)-L_{b}(v)\left(\pi a_{3} u_{2}^{\prime}-a_{2} u_{1}\right)=0, \\
& L_{a}(v)\left(b_{1} u_{1}^{\prime}-\pi b_{1} u_{2}\right)-L_{b}(v)\left(a_{4} u_{1}^{\prime}-\pi a_{1} u_{2}\right)=0 .
\end{aligned}
$$

In fact, expanding the left member of $\left(9_{1}\right)$ and applying (3), (5) and (7), we have,

$$
\begin{aligned}
d_{12} v_{1} u_{1}^{\prime}+\pi d_{21} v_{2} u_{2}^{\prime} & +d_{32} u_{1}^{\prime} v_{1}^{\prime}+\pi d_{31} v_{1}^{\prime} u_{2}^{\prime}+d_{42} u_{1}^{\prime} v_{2}^{\prime}+\pi d_{11} u_{2}^{\prime} v_{2}^{\prime} \\
= & d_{12}+v_{1}^{\prime}\left(d_{12} u_{1}+d_{32} u_{1}^{\prime}+\pi d_{31} u_{2}^{\prime}\right)+d_{21} \\
& +\pi v_{2}^{\prime}\left(d_{21} u_{2}+d_{31} u_{1}^{\prime}+d_{41} u_{2}^{\prime}\right)=0 .
\end{aligned}
$$

The other equations of (9) are proved in a similar manner. Now $L_{u}(v)$ and $L_{\iota}(v)$ are not both zero, and hence the equation

$$
\begin{aligned}
& \left(a_{2} u_{1}^{\prime}+\pi a_{1} u_{2}^{\prime}\right)\left\{B-\left(b_{1} v_{1}+b_{3} v_{1}^{\prime}\right) \int_{x_{1}}^{x_{2}} f P u d x\right\} \\
& -\left(b_{2} u_{1}^{\prime}+\pi b_{1} u_{2}^{\prime}\right)\left\{A-\left(a_{1} v_{1}^{\prime}+a_{3} v_{1}^{\prime}\right) \int_{x_{1}}^{x_{2}} f P u d x\right\}=0
\end{aligned}
$$

is a result of $(8)$ and $\left(9_{1}\right)$. Furthermore, $(8)$ is a result of $\left(9_{1}\right)$ and $(10)$ unless

$$
a_{2} u_{1}^{\prime}+\pi a_{1} u_{2}^{\prime}=0, \quad b_{2} u_{1}^{\prime}+\pi b_{1} u_{2}^{\prime}=0 .
$$


These equations can hold only when either $d_{12}=0$ or $u_{1}^{\prime}=0, u_{2}^{\prime}=0$. But if $u_{1}^{\prime}=0, u_{2}^{\prime}=0$ then $u_{1} \neq 0, u_{2} \neq 0$, for a solution of $\left(1_{0}\right)$ can not vanish together with its derivative, and it follows from $\left(7_{1}\right)$ that $d_{12}=0$. Therefore equation (10) is equivalent to (8) unless $d_{12}=0$. If we expand the terms of (10) we have

$$
\begin{aligned}
\left(a_{2} B-A b_{2}\right) u_{1}^{\prime}+\pi\left(a_{1} B\right. & \left.-A b_{1}\right) u_{2}^{\prime} \\
& -\left(d_{21} v_{1} u_{1}^{\prime}+d_{23} v_{1}^{\prime} u_{1}^{\prime}+\pi d_{13} v_{1}^{\prime} u_{2}^{\prime}\right) \int_{x_{1}}^{x_{2}} f P u d x=0,
\end{aligned}
$$

or, on account of $(5)$ and $\left(7_{2}\right)$,

$$
\left(a_{2} B-A b_{2}\right) u_{1}^{\prime}+\pi\left(a_{1} B-A b_{1}\right) u_{2}^{\prime}+d_{12} \int_{x_{1}}^{x_{2}} f P u d x=0,
$$

an equation which does not contain $v$, and which is equivalent to (8) except when $d_{12}=0$. Three similar equations may be derived from (8) by using $\left(9_{2}\right)$, $\left(9_{3}\right)$ and $\left(9_{4}\right)$. We have, replacing $\pi$ and $P$ by their values, the four equations,

$$
1)\left\{\begin{array}{l}
\left(a_{2} B-A b_{2}\right) u_{1}^{\prime}+e^{\int_{x_{1}}^{x_{2}} p l x}\left(a_{1} B-A b_{1}\right) u_{2}^{\prime}+d_{12} \int_{x_{1}}^{x_{2}} f e^{\int_{x_{1}}^{x} p l x} u d x=0, \\
\left(a_{4} B-A b_{4}\right) u_{1}+e^{\int_{x_{1}}^{x_{2}} p l x}\left(a_{3} B-A b_{3}\right) u_{2}+d_{43} \int_{x_{1}}^{x_{2}} f e^{\int_{x_{1}}^{x} p l x} u d x=0, \\
\left(a_{2} B-A b_{2}\right) u_{1}-e^{\int_{x_{1}}^{x_{2}} p d x}\left(a_{3} B-A b_{3}\right) u_{2}^{\prime}+d_{23} \int_{x_{1}}^{x_{2}} f e^{\int_{x_{1}}^{x}{ }^{p} p l x} u d x=0 \\
\left(a_{4} B-A b_{4}\right) u_{1}^{\prime}-e^{\int_{x_{1}}^{x_{2}} p l x}\left(a_{1} B-A b_{1}\right) u_{2}+d_{14} \int_{x_{1}}^{x_{2}} f e^{\int_{x_{1}}^{x} p d x} u d x=0,
\end{array}\right.
$$

each of which is equivalent to (8) unless the determinant $d_{i k}$ which occurs in it is zero. Since from equation (3) $d_{13}$ and $d_{24}$ vanish together it is easily seen that all the determinants $d_{12}, d_{43}, d_{23}, d_{14}$ cannot be zero unless all determinants $d_{i k}$ are zero, a case to be excluded, since the boundary conditions (2) would be either incompatible or dependent. Therefore :

The necessary and sufficient condition for the existence of a solution of the differential equation

$$
y^{\prime \prime}+p y^{\prime}+q y=f,
$$

under the boundary conditions

$$
\left\{\begin{array}{l}
L_{a}(y) \equiv a_{1} y\left(x_{1}\right)+a_{2} y\left(x_{2}\right)+a_{3} y^{\prime}\left(x_{1}\right)+a_{1} y^{\prime}\left(x_{2}\right)=A, \\
L_{b}(y) \equiv b_{1} y\left(x_{1}\right)+b_{2} y\left(x_{2}\right)+b_{3} y^{\prime}\left(x_{1}\right)+b_{4} y^{\prime}\left(x_{2}\right)=B,
\end{array}\right.
$$

when one solution, $u$, of the homogeneous problem 


$$
y^{\prime \prime}+p y^{\prime}+q y=0, \quad L_{a}(y)=0, \quad L_{b}(y)=0,
$$

exists, but no other solution linearly independent of $u$, is that $A, B$, and $f(x)$ satisfy one of the equations (11) with non-vanishing determinant $d_{i k}$. If $A=0$, $B=0$ the condition is

$$
\int_{x_{1}}^{x_{2}} f e^{\int_{x_{1}}^{x}{ }^{m}{ }^{m l x}} u d x=0 .
$$

If this condition is satisfied there exists a single infinity of solutions of (1) and (2) of the form

$$
y=\bar{y}+c u,
$$

where $\bar{y}$ is any one such solution and $c$ is an arbitr(ary constant.

CAsE II. All minors of $\Delta$ are zero.

In this case the functions $u$ and $v$ are solutions of the homogeneous problem. The determinant $d_{13}$ cannot be zero. For if $d_{13}=0$ then $d_{24}=0$ and since $v$ satisfies the same boundary conditions as $u$ it follows from $\left(7_{1}\right)$ that

$$
\begin{aligned}
& d_{21} u\left(x_{2}\right)+d_{41} u^{\prime}\left(x_{2}\right)=0, \\
& d_{21} v\left(x_{2}\right)+d_{41} v^{\prime}\left(x_{2}\right)=0,
\end{aligned}
$$

and therefore $d_{12}=0, d_{14}=0$, for the determinant $u\left(x_{2}\right) v^{\prime}\left(x_{2}\right)-v\left(x_{2}\right) u^{\prime}\left(x_{2}\right)$ is not zero. From equation $\left(7_{3}\right)$ it follows in the same way that $d_{23}=0, d_{34}=0$ in case $d_{13}=0$, that is, all determinants $d_{i k}$ are zero if $d_{13}$ is zero. We may therefore assume that $d_{13}$ is not zero.

The necessary and sufficient conditions for the existence of a solution of equations (1) and (2) are seen from (6) and the boundary conditions for $u$ and $v$ to be

$$
\begin{gathered}
A-\left(a_{1} v_{1}+a_{3} v_{1}^{\prime}\right) \int_{x_{1}}^{x_{2}} f P u d x+\left(a_{1} u+a_{3} u_{1}^{\prime}\right) \int_{x_{1}}^{x_{2}} f P v d x=0, \\
B-\left(b_{1} v_{1}+b_{3} v_{1}^{\prime}\right) \int_{c_{1}}^{x_{2}} f P u d x+\left(b_{1} u+b_{3} u^{\prime}\right) \int_{x_{1}}^{x_{2}} f P i d x=0,
\end{gathered}
$$

Eliminating each integral in turn we have, on account of (5),

$$
\left\{\begin{array}{l}
\left(a_{1} B-A b_{1}\right) u_{1}+\left(a_{3} B-A b_{3}\right) u_{1}^{\prime}+d_{13} \int_{x_{1}}^{x_{2}} f e^{\int_{x_{1}}^{x} p l x} u d x=0, \\
\left(a_{1} B-A b_{1}\right) v_{1}+\left(a_{3} B-A b_{3}\right) v_{1}^{\prime}+d_{13} \int_{x_{1}}^{x_{2}} f e^{\int_{x_{1}}^{x} p l x} v d x=0 .
\end{array}\right.
$$

Since $d_{13}$ can not be zero in this case, these equations are equivalent to the preceding pair, and hence : 
The equations (13) are the necessary and sufficient conditions for the existence of a solution of the differential equation (1) under the boundary condition (2) in case two linearly independent solutions $u, v$ of the homogeneous problem exist. If $A=0, B=0$ the conditions are

$$
\int_{x_{1}}^{x_{3}} f e^{\int_{x_{1}}^{x_{2}} p d x} n d x=0, \quad \int_{x_{1}}^{x_{2}} f e^{\int_{x_{1}}^{x_{2}} p d x} v d x=0 .
$$

If these conditions are satisfied there exists a doubly infinite set of solutions of (1) and (2) of the form

$$
y=\bar{y}+c u+d v,
$$

where $\bar{y}$ is any one such solution and $c, d$ are arbitrary constants.

\section{\$2. The existence of the first normal functions.}

There exists a solution of the homogeneous problem when and only when the determinant $\Delta$ of equations (6) is zero. If the coefficients of the differential equation contain a parameter $\lambda$, then $\Delta$ is a function of $\lambda$, and $\lambda$ must have a value which is a zero of $\Delta(\lambda)$ in order that a solution of the required type exists. These zeros $\lambda_{i}$ of $\Delta(\lambda)$ will be called normal parameter values, and the corresponding solutions $y_{i}$ of the homogeneous problem normal functions. We shall consider the case that $q$ is a linear function of $\lambda$. The problem to be treated is to prove the existence and investigate the properties of the normal parameter values and normal functions for the differential equation

$$
y^{\prime \prime}+p(x) y^{\prime}+[\lambda A(x)-B(x)] y=0
$$

under the boundary conditions

$$
\left\{\begin{array}{l}
L_{a}(y) \equiv a_{1} y\left(x_{1}\right)+a_{2} y\left(x_{2}\right)+a_{3} y^{\prime}\left(x_{1}\right)+a_{4} y^{\prime}\left(x_{2}\right)=0 \\
L_{b}(y) \equiv b_{1} y\left(x_{1}\right)+b_{2} y\left(x_{2}\right)+b_{3} y^{\prime}\left(x_{1}\right)+b_{4} y^{\prime}\left(x_{2}\right)=0
\end{array}\right.
$$

It will be assumed that the following conditions are satisfied:

$$
\begin{array}{lr}
d_{24}=\pi d_{13} & \left(\pi=e^{J_{x_{1}}^{x_{2}} p l x}\right), \\
B(x) \geqq 0 & \left(x_{1} \leqq x \leqq x_{2}\right),
\end{array}
$$

(16) all the determinants $d_{12}, d_{14}, d_{23}, d_{13}$ that are not zero have the same sign. The following statement is a result of condition (16):

Lemma. The values of the expression

$$
-\left[y y^{\prime} e^{\int_{x_{1}}^{x}{ }^{p l x}}\right]_{x_{1}}^{x_{2}} \equiv y\left(x_{1}\right) y^{\prime}\left(x_{1}\right)-\pi y\left(x_{2}\right) y^{\prime}\left(x_{2}\right)
$$

formed for all functions which satisfy $\left(2_{0}\right)$ are positive or zero. 
Any function $y$ which satisfies $\left(2_{0}\right)$ also satisfies the equations

$$
\begin{aligned}
& d_{21} y\left(x_{2}\right)+d_{31} y^{\prime}\left(x_{1}\right)+d_{41} y^{\prime}\left(x_{2}\right)=0, \\
& d_{12} y\left(x_{1}\right)+d_{32} y^{\prime}\left(x_{1}\right)+\pi d_{31} y^{\prime}\left(x_{2}\right)=0, \\
& d_{13} y\left(x_{1}\right)+d_{23} y\left(x_{2}\right)+d_{13} y^{\prime}\left(x_{2}\right)=0, \\
& d_{14} y\left(x_{1}\right)+\pi d_{13} y\left(x_{2}\right)+d_{34} y^{\prime}\left(x_{1}\right)=0 .
\end{aligned}
$$

Hence

$\left(18_{1}\right) d_{12}\left[y\left(x_{1}\right) y^{\prime}\left(x_{1}\right)-\pi y\left(x_{2}\right) y^{\prime}\left(x_{2}\right)\right]=d_{23}\left[y^{\prime}\left(x_{1}\right)\right]^{2}+2 \pi d_{13} y^{\prime}\left(x_{1}\right) y^{\prime}\left(x_{2}\right)+\pi d_{14}\left\lfloor y^{\prime}\left(x_{2}\right)\right]^{2}$, $\left(18_{2}\right) d_{43}\left[y\left(x_{1}\right) y^{\prime}\left(x_{1}\right)-\pi y\left(x_{2}\right) y^{\prime}\left(x_{2}\right)\right]=d_{14}\left[y\left(x_{1}\right)\right]^{2}+2 \pi d_{13} y\left(x_{1}\right) y\left(x_{2}\right)+\pi d_{23}\left[y\left(x_{2}\right)\right]^{2}$, $\left(1_{3}\right) d_{23}\left[y\left(x_{1}\right) y^{\prime}\left(x_{1}\right)-\pi y\left(x_{2}\right) y^{\prime}\left(x_{2}\right)\right]=d_{12}\left[y\left(x_{1}\right)\right]^{2}+\pi d_{43}\left[y^{\prime}\left(x_{2}\right)\right]^{2}$, $\left(18_{4}\right) d_{14}\left[y\left(x_{1}\right) y^{\prime}\left(x_{1}\right)-\pi y\left(x_{2}\right) y^{\prime}\left(x_{2}\right)\right]=d_{43}\left[y^{\prime}\left(x_{1}\right)\right]^{2}+\pi d_{12}\left[y\left(x_{2}\right)\right]^{2}$.

The discriminants of the right members of $\left(18_{1}\right)$ and $\left(18_{2}\right)$ are

$$
\pi\left(d_{14} d_{23}-\pi d_{13}^{2}\right)=\pi\left(d_{i 4} d_{23}-d_{13} d_{24}\right) \text {. }
$$

The second member when expanded is seen to be equal to

$$
\pi d_{12} d_{43} \text {. }
$$

Since the determinants $d_{i k}$ which occur in the first members of (18) cannot all be zero the truth of the above lemma is seen at once.

Consider now the following minimum problem:

Among all functions $y(x)$ which satisfy the bonndary conditions $\left(2_{0}\right)$ and the equation*

$$
\int_{x_{1}}^{x_{2}} A P y^{2} d x=1 \quad\left(P=e \int_{x_{1}}^{x} p^{p d x}\right),
$$

and are continuous together with their first and second derivatives in the interval $\left(x_{1}, x_{2}\right)$, that one is required which gives the least value to the expression

$$
J=\int_{x_{1}}^{x_{2}}\left(y^{\prime 2}+B y^{2}\right) P d x-\left[y y^{\prime} P\right]_{x_{1}}^{x_{2}} .
$$

The values of $J$ are all positive or zero, on account of conditions (15) and the lemma proved by means of condition (16). Hence there exists a definite lower limit $\lambda_{0}$ of the values of $J$ under the above conditions. It will be proved that

* It is here assumed that $A$ is not everywhere negative in $\left(x_{1}, x_{2}\right)$. This is no restriction on the problem since $\Lambda$ occurs in the equation $\left(1^{\prime}\right)$ multiplied by the parameter $\lambda$. 
this value $\lambda_{0}$ is a normal parameter value for the differential equation $\left(1^{\prime}\right)$ under the boundary conditions $\left(2_{0}\right)$, and that the corresponding normal function is the solution of the minimum problem. If $\lambda_{0}$ were not a normal parameter value there would exist, according to the theorems of $\S 1$, a solution of the differential equation

$$
y^{\prime \prime}+p y^{\prime}+\left(\lambda_{0} A-B\right) y=f^{\prime}
$$

under the boundary conditions $\left(2_{0}\right)$, whatever be $f$. It will be shown that $f$ could be so chosen that the solution of the equation would satisfy all conditions of the minimum problem, and would give to $J$ a value less than the lower limit of all such values, $\lambda_{0}$. The value $\lambda_{0}$ must therefore be a normal parameter value.

Let

$$
u_{1}, u_{2}, u_{3}, \ldots
$$

be an infinite series of approximating functions for the minimum problem, i. e., an infinite series of functions $u_{h}$ which satisfy the equation

$$
\operatorname{limit}_{h=\infty} J\left(u_{h}\right)=\lambda_{0},
$$

and all the conditions of the minimum problem.

LEMma. The approximating functions $u_{h}$ can be so chosen that there exists a fixed number $G$ such that for all $h$

$$
\left|u_{h}(x)\right|<\Theta \quad\left(x_{1} \leqq x \leqq x_{2}\right)
$$

From the inequalities,

$$
\begin{gathered}
\left|\int_{x_{1}}^{x} u_{h}^{\prime} d x\right| \leqq \int_{x_{1}}^{x}\left|u_{h}^{\prime}\right| d x<\int_{x_{1}}^{x}\left(u_{h}^{\prime 2}+1\right) d x, \\
\int_{x_{1}}^{x} u_{h}^{\prime} d x \leqq \frac{1}{m} \int_{x_{1}}^{x} P u_{h}^{\prime 2} d x<\frac{J\left(u_{h}\right)}{m},
\end{gathered}
$$

where $m$ is the minimum of $P=e^{\int_{x_{1}}^{x} p^{\prime l x}}$ in the interval $\left(x_{1}, x_{2}\right)$, it follows that

$$
\left|u_{h}(x)-u_{h}\left(x_{1}\right)\right|<\frac{\lambda_{0}}{m}+\left(x_{2}-x_{1}\right)+\epsilon_{h},
$$

where limit ${ }_{h=\infty} \epsilon_{h}=0$. The functions $u_{h}$ therefore have the form

$$
u_{h}(x)=c_{h}+\gamma_{h}(x),
$$

where $c_{h}$ are constants and $\gamma_{h}$ a series of functions such that the maxima of their absolute values remain under a fixed linit. From the equation 


$$
\int_{x_{1}}^{x_{3}} A P u_{h}^{2} d x=c_{h}^{2} \int_{x_{1}}^{x_{2}} A P d x+2 c_{h} \int_{x_{1}}^{x_{2}} A P \gamma_{h} d x+\int_{x_{1}}^{x_{3}} A P \gamma_{h}^{2} d x=1,
$$

it follows that the quantities $c_{h}$ all lie between finite limits unless

and consequently

$$
\int_{x_{1}}^{x_{2}} A P d x=0, \quad \operatorname{limit}_{h=\infty} \int_{x_{1}}^{x_{2}} A \gamma_{h} d x=0
$$

$$
\operatorname{limit}_{h=\infty} \int_{x_{1}}^{x_{2}} A \gamma_{h}^{2} d x=1 \text {. }
$$

From equation $\left(18_{4}\right)$, which is satisfied by the functions $u_{h}$, we have

$$
d_{43}\left[u_{h}^{\prime}\left(x_{1}\right)\right]^{2}+\pi d_{12}\left[u_{h}\left(x_{2}\right)\right]^{2}=-d_{14}\left[u_{h} u_{h}^{\prime} P\right]_{x_{1}}^{x_{2}} \leqq d_{12} J\left(u_{h}\right) .
$$

Hence, if $\left|c_{h}\right|$ increase beyond limit, $d_{12}=0$ and either $d_{43}=0$, or the values $u_{h}^{\prime}\left(x_{1}\right)$ remain between finite limits. Similarly from $\left(18_{3}\right)$, either $d_{43}=0$, or $u_{h}^{\prime}\left(x_{2}\right)$ remain between finite limits, and hence, from $\left(17_{3}\right)$ and $\left(17_{4}\right)$,

$$
d_{13}+d_{23}=0, \quad d_{14}+\pi d_{13}=0 .
$$

Therefore either $d_{13} \neq 0$ or $d_{34} \neq 0$, for if both are zero then all determinants $d_{i k}$ are zero. Then the boundary conditions $\left(2_{0}\right)$ are equivalent to either $\left(17_{1}\right)$ and $\left(17_{3}\right)$, or to $\left(17_{3}\right)$ and $\left(17_{4}\right)$. Hence, if $\left|c_{h}\right|$ increase beyond limit, the boundary conditions, since $d_{12}$ must be zero, are equivalent to

if $d_{34}=0$, or to

$$
\begin{gathered}
y^{\prime}\left(x_{1}\right)-\pi y^{\prime}\left(x_{2}\right)=0, \\
y\left(x_{1}\right)-y\left(x_{2}\right)=0,
\end{gathered}
$$

$$
\begin{aligned}
d_{13}\left[y\left(x_{1}\right)-y\left(x_{2}\right)\right]+d_{43} y^{\prime}\left(x_{2}\right) & =0, \\
\pi d_{13}\left[y\left(x_{1}\right)-y\left(x_{2}\right)\right]+d_{43} y^{\prime}\left(x_{1}\right) & =0,
\end{aligned}
$$

if $d_{34} \neq 0$. [In either case the boundary conditions are satisfied by $y=$ const., and hence by $\gamma_{h}=u_{h}-c_{h}$ ], and we have also

$$
-\left[\gamma_{h} \gamma_{h}^{\prime} P\right]_{r_{1}}^{r_{2}}=-\left[u_{h} u_{h}^{\prime} P\right]_{x_{1}}^{x_{2}}+c_{h}\left[\pi u_{h}^{\prime}\left(x_{2}\right)-u_{h}^{\prime}\left(x_{1}\right)\right]=-\left[u_{h} u_{h}^{\prime} P\right]_{x_{1}}^{r_{2}} .
$$

Therefore

Let

$$
J\left(\gamma_{h}\right)=J\left(u_{h}\right)
$$

From equation (22)

$$
\delta_{h}=\int_{x_{1}}^{x_{2}} A P \gamma_{h}^{2} d x
$$

$$
\operatorname{limit}_{h=x} \delta_{h}=1 \text {. }
$$


Therefore if $\left|c_{\lambda}\right|$ increase without limit, $i$. e., if the functions $u_{\hbar}$ do not satisfy condition (21) there exists a series of functions

$$
\bar{u}_{h}(x)=\frac{u_{h}(x)-c_{h}}{\sqrt{\left|\delta_{h}\right|}}
$$

which satisfy (21), and since they satisfy the equations

$$
\begin{gathered}
L_{a}\left(\bar{u}_{h}\right)=0, \quad L_{b}\left(\bar{u}_{h}\right)=0, \\
\int_{x_{1}}^{x_{h}} A P \bar{u}_{h}^{2} d x=\frac{1}{\delta_{h}} \int_{x_{1}}^{x_{2}} A P \gamma_{h}^{2} d x=1, \\
\operatorname{limit}_{h=\infty} J\left(\bar{u}_{h}\right)=\operatorname{limit}_{h=\infty} \frac{1}{\delta_{h}} J\left(u_{h}\right)=\lambda_{0},
\end{gathered}
$$

they form a series of approximating functions for the minimum problem. The lemma stated above is therefore proved.

Define an infinite series of functions $f_{h}$ by the equations

$$
\left(u_{h}^{\prime} P\right)^{\prime}+\left(\lambda_{0} A-B\right) P u_{h}=f_{h} .
$$

Multiplying these equations by $u_{n}$ and integrating from $x_{1}$ to $x_{z}$ we have, on account of the condition (19),

$$
\left[u_{h} u_{h}^{\prime} P\right]_{x_{1}}^{x_{2}}-\int_{x_{1}}^{x_{2}}\left(u_{h}^{\prime 2}+B u_{h}^{2}\right) P d x+\lambda_{0}=\int_{x_{1}}^{x_{2}} f_{h} u_{h} d x .
$$

Hence, on account of (20),

$$
\operatorname{limit}_{h=\infty} \int_{x_{1}}^{x_{2}} f_{h} u_{h} d x=0
$$

Under the assumption that $\lambda_{0}$ is not a normal parameter value there exists for each $h$ a solution of the differential equation

$$
U_{h}^{\prime \prime}+p U_{h}^{\prime}+\left(\lambda_{0} A-B\right) U_{h}=A u_{h},
$$

or, multiplying by $P=e^{\int_{x_{1}}^{x} p t x}$, of the equation

$$
\left(U_{h}^{\prime} P\right)^{\prime}+\left(\lambda_{0} A-B\right) P U_{h}=P A u_{h},
$$

under the boundary conditions $\left(2_{0}\right)$. Consider the functions

$$
v_{h}=u_{h}+c U_{h}
$$

where $c$ is a constant. These functions satisfy the boundary conditions $\left(2_{0}\right)$ and the equation

$$
\left(v_{h}^{\prime} P\right)^{\prime}+\left(\lambda_{v} A-B\right) P v_{h}=f_{h}+c P A u_{h} .
$$


Multiplying this equation by $v_{h}=u_{n}+c U_{h}$ and integrating from $x_{1}$ to $x_{2}$ the following equation results :

$$
\begin{aligned}
{\left[v_{h} v_{h}^{\prime} P\right]_{x_{1}}^{x_{3}} } & -\int_{x_{1}}^{x_{3}}\left(v_{h}^{\prime 2}+B v_{h}^{2}\right) P d x+\lambda_{0} \int_{x_{1}}^{x_{h}} A P v_{k}^{2} d x \\
& =\int_{x_{1}}^{x_{2}} f_{h} u_{h} d x+c \int_{x_{1}}^{x_{2}}\left(f_{h} U_{h}+P A u_{h}^{2}\right) d x+c^{2} \int_{x_{1}}^{x_{2}} P A u_{h} U_{h} d x
\end{aligned}
$$

Now from equations (23) and (25) it follows that

$$
\begin{aligned}
\int_{x_{1}}^{x_{h}}\left(f_{h} U_{h}-P A u_{h}^{2}\right) d x=\int_{x_{1}}^{x_{2}}\left\{\left(u_{h}^{\prime} P\right)^{\prime} U_{h}-\left(U_{h}^{\prime} P\right) u_{h}\right\} d x \\
=\left[\left(u_{h}^{\prime} U_{h}-U_{h}^{\prime} u_{h}\right) P\right]_{x_{1}}^{x_{x_{1}}} .
\end{aligned}
$$

The functions $u_{h}$ and $U_{h}$ satisfy the boundary conditions $\left(2_{0}\right)$ and hence equations (17). Since $P\left(x_{1}\right)=1, P\left(x_{2}\right)=\pi$, it follows from $\left(17_{1}\right)$ and $\left(17_{2}\right)$ that

$$
\begin{aligned}
d_{12}\left[\left(u_{h}^{\prime} U_{h}-U_{h}^{\prime} u_{h}\right) P\right]_{x_{1}}^{x_{2}}=\pi u_{h}^{\prime}\left(x_{2}\right) & {\left[d_{31} U_{h}^{\prime}\left(x_{1}\right)+d_{41} U_{h}^{\prime}\left(x_{2}\right)\right] } \\
-\pi U_{h}^{\prime}\left(x_{2}\right)\left[d_{31} u_{h}^{\prime}\left(x_{1}\right)+d_{41} u_{h}^{\prime}\left(x_{2}\right)\right]-u_{h}^{\prime}\left(x_{1}\right)\left[d_{23} U_{h}^{\prime}\left(x_{1}\right)+\pi d_{13} U_{h}^{\prime}\left(x_{2}\right)\right] & \\
& +U_{h}^{\prime}\left(x_{1}\right)\left[d_{23} u_{h}^{\prime}\left(x_{1}\right)+\pi d_{13} u_{h}^{\prime}\left(x_{2}\right)\right]=0 .
\end{aligned}
$$

Hence

$$
\left[\left(u_{h}^{\prime} U_{h}-U_{h}^{\prime} u_{h}\right) P\right]_{x_{1}}^{x_{2}}=0
$$

unless $d_{12}=0$. By using other pairs of equations (17) it is seen that this equation must hold in any case, since all the determinants $d_{i k}$ cannot be zero. Therefore

Since

$$
\int_{x_{1}}^{x_{2}}\left(f_{h} u_{h}-P A u_{h}^{2}\right) d x=0 .
$$

equation (26) becomes

$$
\int_{x_{1}}^{x_{2}} P A u_{h}^{2} d x=1
$$

$$
\lambda_{\uparrow} \int_{x_{1}}^{x_{2}} P A v_{h}^{2} d x-J\left(v_{h}\right)=\int_{x_{1}}^{x} f_{h} u_{h} d x+2 c+c^{2} \int_{x_{1}}^{x_{2}} P A u_{h} U_{h} d x .
$$

Now from equation (4)

$$
U_{h}=c_{h} u+d_{h} v+u \int_{x_{1}}^{x} P A u_{h} v d x+v \int_{x}^{x_{2}} P A u_{h} u d x,
$$

where $u, v$ are the linearly independent solutions of the equation

$$
y^{\prime \prime}+p y^{\prime}+(\lambda A-B) y=0
$$


and $c_{h}, d_{h}$ are constants whose values are so determined that $U_{h}$ satisfies the boundary conditions $\left(2_{0}\right)$. Referring to equations $(6)$ one sees that $c_{h}$ and $d_{h}$ are linear combinations of the two integrals

$$
\int_{x_{1}}^{x_{2}} P A u_{h} u d x, \quad \int_{x_{1}}^{x_{2}} P A u_{h} v d x,
$$

the coefficients being constants independent of $h$. Therefore since the functions $u_{h}$ satisfy condition (20) it follows that the functions $U_{h}$ satisfy a similar condition, and that the quantities

$$
\left|\int_{x_{1}}^{x_{9}} P A u_{h} U_{h} d x\right|
$$

which occur in (27) remain for all $h$ under some fixed limit $B$. Then from (27)

$$
\lambda_{0} \int_{x_{1}}^{x_{3}} P A v_{h}^{2} d x-J\left(v_{h}\right)>\int_{x_{1}}^{x_{2}} f_{h} v_{h} d x+2 c-c^{2} B .
$$

Let $c$ be chosen as a positive number so small that

$$
\text { - } 2 c-c^{2} B>0 \text {. }
$$

Write $2 c-c^{2} B=\delta$. It follows from (24) that $h$ may be taken so large that

$$
\int_{x_{1}:}^{x_{2}} f_{h} u_{h} d x<\delta
$$

Then for this value of $h$ we have

$$
\lambda_{0} \int_{1 / 1}^{x_{2}} P A v_{h}^{2} d x-J\left(v_{h}\right)>0 .
$$

Therefore

and

$$
\int_{x_{1}}^{x_{2}} P A v_{h}^{2} d x>0
$$

is a real number. Let

$$
\alpha=\left\{\int_{x_{1}}^{x_{2}} P A v_{h}^{2} d x\right\}^{1}
$$

$$
y=\stackrel{v_{h}}{a}
$$

This function satisfies all conditions of the mininum problem. It must therefore give to $J$ a value less than or equal to $\lambda_{0}$, the lower limit of the values of $J$ under the conditions of the problem. But dividing (28) by $\alpha^{2}$ we have

$$
\lambda_{0}-\frac{1}{\alpha^{2}} J\left(v_{h}\right)=\lambda_{0}-J(y)>0,
$$


which is impossible. The assumption that $\lambda_{0}$ is not a normal parameter value therefore leads to a contradiction. Hence the lower limit $\lambda_{0}$ is a normal parameter value, and there exists a normal function $y_{0}$, not identically zero, which satisfies the differential equation

$$
y_{0}^{\prime \prime}+p y_{0}^{\prime}+\left(\lambda_{0} A-B\right) y_{0}=0
$$

and the boundary conditions $\left(2_{0}\right)$.

Multiplying equation (29) by $P y_{0}$ and integrating from $x_{1}$ to $x_{2}$, we derive the equation

$$
\lambda_{0} \int_{x_{1}}^{x_{2}} P A y_{0}^{2} d x=J\left(y_{0}\right) .
$$

Since $\lambda_{0}$ is positive or zero the integral in the first member is positive unless $J\left(y_{0}\right)=0$, i. e., unless $y_{0}$ is a constant. Hence the arbitrary constant factor of $y_{0}$ can be so determined that

$$
\int_{x_{1}}^{x_{2}} P A y_{0}^{2} d x=1
$$

unless $y_{0}=$ const. Even if $y_{0}=$ const. this determination may be made, unless

$$
\int_{x_{1}}^{x_{2}} P A d x=0
$$

for if the integral is not zero it may be assumed without restriction to be positive, as the function $A$ occurs in the differential equation multiplied by the parameter $\lambda$. Now equation (32) cannot hold if $y_{0}=$ const., since if (32) is satisfied $\lambda_{0}$ must be greater than zero. In fact, since the approximating functions $u_{h}$ satisfy the equation

$$
\int_{x_{1}}^{x_{2}} P A u_{h}^{2} d x=1
$$

it follows that if (32) holds, each function $u_{h}$ must assume for some two values $x=x_{h}, x=\bar{x}_{h}$ two values $u_{h}\left(x_{h}\right), u_{h}\left(\bar{x}_{h}\right)$ which differ by an amount equal to $\delta$, a fixed positive number independent of $h$. Let $Y_{h}$ represent the ordinate of the straight line connecting the two points $\left[x_{h}, u_{h}\left(x_{h}\right)\right]$ and $\left[\bar{x}_{h}, u_{h}\left(\bar{x}_{h}\right)\right]$. Then

Now

$$
\left|Y_{h}^{\prime}\right|=\frac{\delta}{\bar{x}_{h}-x_{h}}, \quad Y^{\prime \prime}=0 .
$$

Write

$$
J\left(u_{h}\right) \geqq \int_{x_{h}}^{\bar{x}_{h}} u_{h}^{\prime 2} d x .
$$

$$
\eta_{h}=Y_{h}-u_{h} \text {. }
$$


Then since $\eta_{h}$ vanishes at $x_{h}$ and at $\bar{x}_{h}$, and $Y^{\prime \prime}=0$,

$$
\int_{x_{h}}^{\bar{x}_{h}} u_{h}^{\prime 2} d x=\int_{x_{h}}^{\bar{x}_{h}}\left(Y_{h}^{\prime}-\eta_{h}^{\prime}\right)^{2} d x=\frac{\delta^{2}}{\bar{x}_{h}-x_{h}}+\int_{x_{h}}^{\bar{x}_{h}} \eta_{h}^{\prime 2} d x .
$$

Therefore, for all values of $h$,

anc' $\lambda_{0}$ cannot be zero.

$$
J\left(u_{h}\right)>\frac{\delta^{2}}{x_{2}-x_{1}},
$$

It may therefore be assumed without restriction that the normal function $y_{0}$ satisfies equation (31). Hence from (30) $y_{0}$ gives to $J$ the value $\lambda_{0}$. The normal function $y_{0}$ is therefore the solution of the minimum problem.

§3. The existence of an infinite series of normal functions.

We proceed by complete induction. Suppose that there exist $n$ positive or zero normal parameter values

$$
\lambda_{0}, \lambda_{1}, \cdots, \lambda_{n-1}
$$

and $n$ linearly independent normal functions

$$
y_{0}, y_{1}, \cdots, y_{n-1}
$$

satisfying the differential equations

$$
y_{i}^{\prime \prime}+p y_{i}^{\prime}+\left(\lambda_{i} A-B\right) y_{i}=0
$$

and the boundary conditions $\left(2_{0}\right)$. At most two of the values $\lambda_{i}$ can coincide, since the functions $y_{i}$ are assumed to be linearly independent. Multiplying the differential equation by $P y_{i}$ and integrating from $x_{1}$ to $x_{2}$, we have

$$
\lambda_{i} \int_{x_{1}}^{x_{2}} P A y_{i}^{2} d x=J\left(y_{i}\right) .
$$

The arbitrary constant factor of $y_{i}$ may therefore be so determined that

$$
\int_{x_{1}}^{x_{3}} P A y_{i}^{2} d x=1
$$

except possibly when $J\left(y_{i}\right)=0$, since $\lambda_{i}$ and $J\left(y_{i}\right)$ are positive or zero. If $J\left(y_{i}\right)=0$ then $y_{i}$ is a constant and $\lambda_{i}=0$; hence $y_{i}=y_{0}$, and it has been shown above that $y_{0}$ satisfies (31). Furthermore, from the equations

$$
\begin{aligned}
& \left(P y_{i}^{\prime}\right)^{\prime}+\left(\lambda_{i} A-B\right) y_{i}=0, \\
& \left(P y_{k}^{\prime}\right)^{\prime}+\left(\lambda_{k} A-B\right) y_{k}=0,
\end{aligned}
$$


follows

$$
\left(\lambda_{i}-\lambda_{k}\right) \int_{x_{1}}^{x_{2}} P A y_{i} y_{k} d x=\left[P\left(y_{k}^{\prime} y_{i}-y_{i}^{\prime} y_{k}\right)\right]_{._{1}}^{r_{2}} .
$$

Since $y_{i}$ and $y_{k}$ satisfy the boundary conditions $\left(2_{0}\right)$, the right member is zero,* and hence if $\lambda_{i} \neq \lambda_{k}$,

$$
\int_{x_{1}}^{x_{2}} P A y_{i} y_{k} d x=0
$$

If $\lambda_{i}=\lambda_{k}$ then the functions $y_{i}$ and $y_{k}$ are not uniquely determined. The function $y_{k}$ has the form

$$
y_{k}=Y_{k}+c y_{i},
$$

where $c$ is an arbitrary constant. The value of $c$ may be determined so that

$$
\int_{x_{1}}^{x_{2}} P A y_{i} y_{k} d x=\int_{x_{1}}^{x_{2}} P A y_{i} Y_{k} d x+c=0
$$

It may therefore be assumed without restriction that the normal functions $y_{i}$ satisfy the equations

$$
\begin{array}{r}
\int_{x_{1}}^{x_{2}} P A y_{i}^{2} d x=1 \\
\int_{x_{1}}^{x_{2}} P A y_{i} y_{k} d x=0
\end{array}
$$

To prove the existence of a normal function $y_{n}$ linearly independent of $y_{0}, y_{1}, \cdots, y_{n-1}$, consider the following minimum problem:

It is required to minimize the expression

under the conditions

$$
J(u)=\int_{x_{1}}^{x_{2}}\left(y^{\prime 2}+B y^{2}\right) P d x-\left[P y y^{\prime}\right]_{x 1}^{x_{2}}
$$

$$
\begin{aligned}
& L_{a}(y)=0, \quad L_{b}(y)=0, \\
& \int_{x_{1}}^{x_{2}} P A y^{2} d x=1, \\
& \int_{x_{1}}^{x_{2}} P A y_{i} y d x=0 \quad(i=0,1,2, \cdots, n-1) .
\end{aligned}
$$

Under the conditions (15), (16) there exists a finite lower limit $\lambda_{n}$ of the values of $J$, and an infinite series of approximating functions

$$
u_{1}, u_{2}, u_{3}, \ldots
$$

\footnotetext{
* The proof is the same as that given in the reduction of equation (26) to (27).
}

Am. Math. Soc. 24 
which satisfy all the conditions of the minimum problem and the equation

$$
\operatorname{limit}_{h=\infty} J\left(u_{k}\right)=\lambda_{n} \text {. }
$$

Define as before an infinite series of functions $f_{h}$, by the equations

$$
\left(P u_{h}^{\prime}\right)^{\prime}+\left(\lambda_{n} A-B\right) P u_{h}=f_{h} \text {. }
$$

Under the assumption that for $\lambda=\lambda_{n}$ there exists no solution of $\left(1^{\prime}\right)$ and $\left(2_{0}\right)$ which is linearly independent of $y_{0}, y_{1}, \cdots, y_{n-1}$, there must exist, for each value of $h$, a solution of the equation

$$
U_{h}^{\prime \prime}+p U_{h}^{\prime}+\left(\lambda_{n} A-B\right) U_{h}=A u_{h}
$$

under the boundary conditions $\left(2_{0}\right)$. For by the theorems of $\S 1$ the equations

$$
\int_{x_{1}}^{r_{2}} A y_{i} u_{h} d x=0, \int_{x_{1}}^{x_{2}} A y_{k} u_{h} d x=0 \quad(h=1,2,3, \cdots),
$$

which are satisfied by all the functions $u_{k}$, are sufficient conditions for the existence of such solutions $U_{k}$ in case $\lambda_{n}=\lambda_{i}=\lambda_{k}$; the first equation alone is the sufficient condition in case $\lambda_{n}$ is equal to but one of the normal values $\lambda_{i}$; and there is no condition necessary in case $\lambda_{n}$ is not a normal value. The possibility that $\lambda_{n}$ is a normal parameter value different from $\lambda_{0}, \lambda_{1}, \ldots, \lambda_{n-1}$ is excluded by the assumption that for $\lambda=\lambda_{n}$ no solution of $\left(1^{\prime}\right)$ and $\left(2_{0}\right)$ exists which is linearly independent of $y_{0}, y_{1}, \ldots, y_{n-1}$.

Since

$$
\int_{x_{1}}^{x_{2}} P A y_{i} u_{h} d x=0 \quad\left(\begin{array}{l}
i=0,1, \cdots, n-1 \\
h=1,2,3, \cdots
\end{array}\right),
$$

it follows from the equations

that

$$
\begin{gathered}
\left(P y_{i}^{\prime}\right)^{\prime}+\left(\lambda_{i} A-B\right) P y_{i}=0, \\
\left(P U_{h}^{\prime}\right)^{\prime}+\left(\lambda_{n} A-B\right) P U_{h}=P A u_{h},
\end{gathered}
$$

$$
\left(\lambda_{n}-\lambda_{i}\right) \int_{x_{1}}^{x_{9}} P A y_{i} U_{h} d x=\left[P\left(y_{i} U_{h}-U_{h}^{\prime} y_{i}\right)\right]_{x_{1}}^{x_{9}} \text {. }
$$

The right member is zero since $y_{i}$ and $U_{h}$ both satisfy the boundary conditions $\left(2_{0}\right)$. Hence if $\lambda_{n} \neq \lambda_{i}$ the functions $v_{h}$ satisfy the equations

$$
\int_{x_{1}}^{x_{3}} A y_{i} U_{h} d x=0
$$


If $\lambda_{n}=\lambda_{i}$ then the functions $U_{h}$ are not uniquely determined by (37) and (2 $\left.2_{0}\right)$, but have the form

$$
U_{h}=\bar{U}_{h}+c_{n} y_{i},
$$

where $c_{n}$ are arbitrary constants. These constants may be so determined that

$$
\int_{x_{1}}^{x_{2}} P A y_{i} U_{h} d x=\int_{x_{1}}^{x_{2}} P A y_{i} U_{h} d x+c_{h}=0 \text {. }
$$

The functions $U_{h}$, and therefore the functions

$$
v_{h}=u_{h}+c U_{h},
$$

where $c$ is a constant, satisfy all the conditions of the minimum problem except

$$
\int_{r_{1}}^{x_{2}} P A v_{h}^{2} d x=1 .
$$

The assumption, that for $\lambda=\lambda_{n}$ no normal function linearly independent of $y_{0}, y_{1}, \cdots, y_{n-1}$ exists, may now be shown to be false by the method used in the case of the first normal function. There exists $n+1$ normal functions provided $n$ exist, and the existence of a first has been proved. Hence an infinite series of normal functions $y_{n}$ exists, and an infinite series of corresponding normal parameter values $\lambda_{n}$ which are defined as the lower limits of the expression $J(y)$ under the conditions $\left(2_{0}\right),(35),(36)$.

As has been shown above, it may be assumed without restriction that these functions satisfy the equations

$$
\begin{array}{rlrl}
\int_{x_{1}}^{x_{2}} P A y_{n} y_{m} d x & =0 & (n+m), \\
\int_{x_{1}}^{x_{8}} P A y_{n}^{2} d x & =1 .
\end{array}
$$

The function $y_{n}$ therefore satisfies the conditions of the minimum problem used to define $\lambda_{n}$. Multiplying the equation

$$
\left(P y_{n}^{\prime}\right)^{\prime}+\left(\lambda_{n} A-B\right) P y_{n}=0
$$

by $y_{n}$ and integrating from $x_{1}$ to $x_{2}$, we have

$$
J\left(y_{n}\right)=\lambda_{n} \text {. }
$$

Hence $y_{n}$ is the solution of the minimum problem. From the definition of $\lambda_{n}$ as the lower limit of $J$ under the conditions, it may be seen without difficulty that the series $y_{n}$ contains all normal functions whose corresponding parameter value $\lambda_{n}$ is positive or zero. 
The values $\lambda_{n}$ increase without limit, for they are the zeros of $\Delta$, which is an integral transcendental function of $\lambda$.* $^{*}$ If these zeros had a limiting value $\bar{\lambda}$ then $\Delta$ would vanish for $\lambda=\bar{\lambda}$ together with all its derivatives.

If $A$ changes sign in the interval $\left(x_{1}, x_{2}\right)$ the above method, with the single alteration that the equation

be replaced by

$$
\int_{x_{1}}^{x_{2}} P A y^{2} d y=1
$$

$$
\int_{x_{1}}^{x_{2}} P A y^{2} d x=-1 \text {, }
$$

shows the existerce of an infinite series of negative normal parameter values $\lambda_{i}$ and corresponding normal functions $y_{i}$. The results may be summarized in the following

TheOREM. There exists an infinite sernes of normal parameter values $\lambda_{n}$ and corresponding normal functions $y_{n}$ for the differential equation

$$
y^{\prime \prime}+p(x) y^{\prime}+[\lambda A(x)-B(x)] y=0
$$

under the boundary conditions

$$
\left\{\begin{array}{l}
a_{1} y\left(x_{1}\right)+a_{2} y\left(x_{2}\right)+a_{3} y^{\prime}\left(x_{1}\right)+a_{4} y^{\prime}\left(x_{2}\right)=0 \\
b_{1} y\left(x_{1}\right)+b_{2} y\left(x_{2}\right)+b_{3} y^{\prime}\left(x_{1}\right)+b_{4} y^{\prime}\left(x_{2}\right)=0
\end{array}\right.
$$

where, in writing $a_{i} b_{k}-a_{k} b_{i}=d_{i k}$,

$$
e^{\int_{x_{1}}^{x_{2}} p d x} d d_{13}=d_{24}
$$

and where all the determinants $d_{12}, d_{14}, d_{23}, d_{43}$ which are not zero have the same sign.

If the function $A(x)$ is not negative in the interval $\left(x_{1}, x_{2}\right)$ the values $\lambda_{i}$ are not negative, and increase without limit with $i$. If $A(x)$ changes sign in the interval the values $\lambda_{i}$ include an infinite series of positive quantities increasing without limit and an infinite series of negative quantities decreasing without limit.

The function $y_{n}$ minimizes the expression

under the conditions

$$
J(y)=\int_{x_{1}}^{x_{2}}\left(y^{\prime 2}+B y^{2}\right) P d x-\left[y y^{\prime} P\right]_{x_{1}}^{x_{2}}
$$

$$
L_{a}(y)=0, \quad L_{b}(y)=0,
$$

* See PiCaRD, Traité d'analyse, t. 3 (1896), p. 92. 


$$
\begin{aligned}
& \int_{x_{1}}^{x_{2}} P A y^{2} d x= \pm 1, \\
& \int_{x_{1}}^{x_{3}} P A y_{i} y d x=0 \quad[i=0, \pm 1, \pm 2, \cdots, \pm(n-1)],
\end{aligned}
$$

and gives to $J$ the value $\pm \lambda_{n}$, the upper signs being taken if $\lambda_{n}$ is positive, the lower signs if $\lambda_{n}$ is negative.

§4. An oscillation theorem for the periodic solutions.

If the coefficients of $\left(1^{\prime}\right)$ are periodic functions with the period $x_{2}-x_{1}$ then any solution of $\left(1^{\prime}\right)$ under the boundary conditions

$$
\begin{array}{r}
y\left(x_{1}\right)-y\left(x_{2}\right)=0, \\
y^{\prime}\left(x_{1}\right)-y^{\prime}\left(x_{2}\right)=0,
\end{array}
$$

is likewise periodic with the period $x_{2}-x_{1}$. In this case, since $d_{12}=0$, $d_{14}=-1, d_{23}=-1, d_{43}=0$, conditions (16) are satisfied. Since $d_{13}=1$, $d_{24}=1$ we must have

$$
\pi=e^{\int_{x_{1}}^{x_{2}} p d x}=1,
$$

or

$$
\int_{x_{1}}^{x_{2}} p d x=0
$$

in order to satisfy condition (3). It will be assumed that this condition is satisfied. Then there exists an infinite series of periodic solutions $y_{n}$ of $\left(1^{\prime}\right)$ when the coefficients are periodic. We shall investigate the number of times $y_{n}$ vanishes in the interval $\left(x_{1}, x_{2}\right)$ under the assumption that

$$
A(x) \geqq 0 \quad\left(x_{1} \leqq x \leqq x_{2}\right) .
$$

Let $\mu_{n}$ and $u_{n}$ denote the normal parameter values and functions for the boundary condition

$$
y\left(x_{1}\right)=0, \quad y\left(x_{2}\right)=0 .
$$

Then, under the assumption $A \geqq 0$, according to STURm's theorems* the function $u_{n}$ vanishes $n$ times within the interval $\left(x_{1}, x_{1}\right)$. We shall determine the number of times the periodic function $y_{n}$ vanishes in $\left(x_{1}, x_{2}\right)$ by means of this fact.

It may be proved from the definition of the normal parameter values as minimal values that

$$
\lambda_{n} \leqq \mu_{n} \leqq \lambda_{n+1}
$$

\footnotetext{
* See BôcHER, Encyklopädie der mathematischen Wissenschaften, II A 7a.
} 
To show that $\mu_{n} \leqq \lambda_{n+1}$ it suffices to form a function $w$ which satisfies the conditions

and gives to

$$
\begin{aligned}
w\left(x_{1}\right) & =0, \quad w\left(x_{2}\right) & =0 \\
\int_{x_{1}}^{x_{2}} P A w^{2} d x & =1, \quad \int_{x_{1}}^{x_{2}} P A u_{i} w d x & =0 \quad(i=0,1,2, \cdots, n-1),
\end{aligned}
$$

$$
\int_{x_{1}}^{x_{2}}\left(w^{\prime 2}+B w^{2}\right) P d y
$$

a value less than or equal to $\lambda_{n+1}$. Consider the function

$$
w=c_{0} y_{0}+c_{1} y_{1}+\cdots+c_{n+1} y_{n+1},
$$

formed from the periodic solutions $y_{i}$. Since $w\left(x_{1}\right)=w\left(x_{2}\right)$ the above boundary condition will be satisfied if $w\left(x_{1}\right)=0$. Now the $n+2$ constants $c_{i}$ may always be so determined that they are not all zero, and that the $n+1$ equations

$$
\begin{aligned}
w\left(x_{1}\right) & =\Sigma c_{k} y_{k}\left(x_{1}\right)=0 \\
\int_{x_{1}}^{x_{2}} P A u_{i} w d x & =\Sigma c_{k} \int_{x_{1}}^{x_{2}} P A u_{i} y_{k} d x=0 \quad(i=0,1, \cdots, n-1)
\end{aligned}
$$

are satısfied, and furthermore so that

$$
\int_{x_{1}}^{x_{2}} P A w^{2} d x=\Sigma c_{k}^{2}=1
$$

Then $w$ satisfies all the conditions of the minimum problem used in defining $\mu_{n}$.

Multiplying the equation

$$
\left(P y_{i}^{\prime}\right)^{\prime}+\left(\lambda_{i} A-B\right) P y_{i}=0
$$

by $y_{k}$, integrating from $x_{1}$ to $x_{2}$ and remembering the conditions which $y_{n}$ satisfies, we have

Hence

$$
-\int_{x_{1}}^{x_{2}} P y_{i}^{\prime} y_{k}^{\prime} d x-\int_{x_{1}}^{x_{2}} P B y_{i} y_{k} d x=0
$$

$$
\int_{x_{1}}^{x_{2}}\left(w^{\prime 2}+B w^{2}\right) P d x=\Sigma c_{k^{2}} \int_{x_{1}}^{x_{2}}\left(y_{k}^{\prime 2}+B y_{k}^{2}\right) P d x=\Sigma c_{k}^{2} \lambda_{k} \leqq \lambda_{n+1} \Sigma c_{k}^{2}=\lambda_{n+1} .
$$

Therefore

To prove the inequality

$$
\mu_{n} \leqq \lambda_{n+1} \text {. }
$$

$$
\lambda_{n} \leqq \mu_{n},
$$


it is to be noticed that $\lambda_{n}$ is the lower limit of the values of

under the conditions

$$
J(y)=\int_{x_{1}}^{x_{2}}\left(y^{\prime 2}+B y^{2}\right) P d x
$$

$$
\begin{gathered}
\int_{x_{1}}^{x_{2}} P A y^{2} d x=1, \quad \int_{x_{1}}^{x_{2}} P A y_{i} y d x=0 \quad(i=0,1,2, \cdots, n-1), \\
y\left(x_{1}\right)=y\left(x_{2}\right), \quad y^{\prime}\left(x_{1}\right)=y^{\prime}\left(x_{2}\right),
\end{gathered}
$$

and since $J(y)$ contains $y^{\prime}$ only in the integral this lower limit will be unchanged when the condition $y^{\prime}\left(x_{1}\right)=y^{\prime}\left(x_{2}\right)$ is omitted.

The function

$$
w=c_{0} u_{0}+c_{1} u_{1}+\cdots+c_{n} u_{n}
$$

satisfies the equation $w\left(x_{1}\right)=w\left(x_{2}\right)$ since it vanishes at $x_{1}$ and $x_{2}$. Then the $n+1$ constants $c_{i}$ can always be determined so that they are not all zero, and that the $n$ equations hold:

$$
\Sigma c_{k} \int_{x_{1}}^{x_{2}}\left(P A y_{i} u_{k} d x=0 \quad(i=0,1,2, \cdots, n-1) .\right.
$$

The common factor of the constants $c_{i}$ may then be determined so that

$$
\int_{x_{1}}^{x_{2}} P A w^{2} d x=\Sigma c_{k}^{2}=1,
$$

and the desired inequality follows as in the preceding case.

Suppose $y$ and $\bar{y}$ are functions satisfying the equations

$$
\begin{aligned}
& \left(P y^{\prime}\right)^{\prime}+(\lambda A-B) P y=0, \\
& \left(P \bar{y}^{\prime}\right)^{\prime}+(\bar{\lambda} A-B) P \bar{y}=0 .
\end{aligned}
$$

Then, since $P\left(x_{1}\right)=1, P\left(x_{2}\right)=1$,

$$
\left[\bar{y} y^{\prime}-y \bar{y}^{\prime}\right]_{a}^{b}=(\bar{\lambda}-\lambda) \int_{a}^{b} A P y \bar{y} d x .
$$

Suppose $a$ and $b$ are consecutive zeros of $\bar{y}$, and that $\bar{y}$ is positive between $a$ and $b$. Then $\bar{y}^{\prime}(a)>0, \bar{y}^{\prime}(b)<0$, for $\bar{y}^{\prime}$ cannot vanish with $\bar{y}$. Suppose further that $\lambda>\bar{\lambda}$ and write $(+)$ for a positive function or constant. Then, since $A>0$,

$$
(+) y(a)+(+) y(b)=-(+) \int_{a}^{b}(+) y d x .
$$

Hence $y$ must vanish between $a$ and $b$. That is, between two zeros of a solu- 
tion of $\left(1^{\prime}\right)$ for a value $\bar{\lambda}$ there lies at least one zero of a solution of $\left(1^{\prime}\right)$ for $\lambda>\bar{\lambda} . *$

Suppose now that $\lambda_{i}=\mu_{n}$. If $y_{i}$ and $u_{n}$ are linearly independent, then $y_{i}$ does not vanish at $x_{1}$ or at $x_{2}$ and since by the well known STurm's theorem the zeros of two linearly independent solutions of the same differential equation of type $\left(1^{\prime}\right)$ separate each other $y_{i}$ vanishes $n+1$ times within the interval $\left(x_{1}, x_{2}\right)$. If $y_{i}$ and $u_{n}$ are linearly dependent $y_{i}$ vanishes with $u_{n}$, that is, $n+1$ times in the interval $\left(x_{1} \leqq x<x_{2}\right)$. But $y_{i}$ is periodic and therefore $\lambda_{i}=\mu_{n}$ only when $n$ is an odd number. Therefore we have from the inequalities $\lambda_{n} \leqq \mu_{n} \leqq \lambda_{n+1}$,

$$
\mu_{2 m-2}<\lambda_{2 m-1} \leqq \mu_{2 m-1} \leqq \lambda_{2 m}<\mu_{2 m} .
$$

If $\lambda_{2 m-1}=\mu_{2 m-1}$ then $y_{2 m-1}$ vanishes $2 m$ times in the interval $\left(x_{1} \leqq x<x_{2}\right)$, as was seen above. If $\lambda_{2 m-1}<\mu_{2 m-1}$ then, since between each pair of zeros of $y_{2 m-1}$ one zero of $u_{2 m-1}$ must lie, $y_{2 m-1}$ vanishes at most $2 m$ times in $\left(x_{1} \leqq x<x_{2}\right)$. But since $\mu_{2 m-2}<\lambda_{2 m-1}, y_{2 m-1}$ vanishes at least $2 m-1$ times in $\left(x_{1} \leqq x<x_{2}\right)$, and since $y_{2 m-1}$ is periodic it follows that $y_{2 m-1}$ vanishes in all cases $2 m$ times for $x_{1} \leqq x<x_{2}$.

If $\lambda_{2 m}=\mu_{2 m-1}$ then $y_{2 m}$ vanishes $2 m$ times in $\left(x_{1} \leqq x<x_{2}\right)$. If $\mu_{2 m-1}<\lambda_{2 m}$ then $y_{2 m}$ has at least $2 m$ zeros in $\left(x_{1} \leqq x<x_{2}\right)$. But since $\lambda_{2 m}<\mu_{2 m}, y_{2 m}$ has at most $2 m+1$ zeros in this interval, and since $y_{2 m}$ is periodic it follows that it vanishes in all cases exactly $2 m$ times for $x_{1} \leqq x<x_{2}$. These results may be summarized in the following

Theorem. There exists an infinite series of normal parameter values $\lambda_{n}$ and corresponding periodic functions $y_{n}$ with the period $x_{2}-x_{1}$, which satisfy the differential equations

where

$$
y_{n}^{\prime \prime}+p y_{n}^{\prime}+\left(\lambda_{n} A-B\right) y_{n}=0
$$

$$
\int_{x_{1}}^{x_{2}} p d x=0, \quad B \geqq 0 \text {, }
$$

and where $p, A, B$ are periodic with the period $x_{2}-x_{1}$. If $A(x) \geqq 0$ in the interval $\left(x_{1}, x_{2}\right)$ the functions $y_{2 m}$ and $y_{2 n-1}$ vanish exactly $2 m$ times for $x_{1} \leqq x<x_{2}$.

Shefrield Scientific School of YaLE University.

* This is a well known result of STURM. 